\title{
CONDITIONING IN ARABIDOPSIS THALIANA
}

\author{
1. HILL \\ A.R.C. Unit of Biometrical Genetics, Department of Genetics, \\ University of Birmingham
}

Received I3.vii.61

The importance of the environment in determining the phenotype of an individual has been known since Johannsen's (1909) experiments with Phaseolus vulgaris, in which he demonstrated that the phenotype expressed was the result of the interaction between genotype and environment. Recently, however, it has been realised that the effects of the environment may in fact be more persistent. Where this persistence is complete, the progeny from individuals which themselves expressed a characteristic phenotype under a given set of environmental conditions, exhibit this phenotype even after the removal of these specific conditions. Such progeny are then said to have been " conditioned ". The most outstanding example of this has been found in an inbred line of flax by Durrant (1958) and Durrant and Tyson (1960) who used all possible combinations of nitrogen, phosphorous and potassium fertilisers as the conditioning treatments. Results of this nature have obvious agricultural implications and it is therefore desirable to determine which plants can be similarly conditioned.

Arabidopsis thaliana, an automatically self-pollinated ephemeral of widespread distribution in waste places throughout the British Isles, was the species chosen for a series of experiments designed to test whether fertiliser alone or in combination with photoperiodic treatment could induce any permanent effect.

Using fertiliser alone, 20 pots of a highly inbred strain were sown in each of 2 treatments, one being John Innes potting compost with all nutrients added. The other was without nutrients until it was found that 8 of the 20 plants within this group were dying, whereupon $5 \mathrm{mls}$. of $\mathrm{o} \cdot \mathrm{or}$ concentration of Langridge's solution (1957) was supplied to these plants. All plants in this and subsequent experiments were recorded for flowering-time, leaf number, rosette diameter and final height.

Of the 40 initially treated plants, 16 (8 from each treatment) were selected for further experiments. These 8 plants from within each treatment were then further subdivided according to the degree of expression of the characters recorded into high, medium and low. Prior to sowing, all seed from the selected lines, together with seed from the original race to be used as controls, was subjected to two cycles of temperature treatment to break its dormancy. The experiment, which was laid down in a glasshouse, was of a randomised block design with six replicate-blocks. Nutrient status and day length were the same for all plants. Re-randomisation was carried out at regular intervals in order to distribute edge effects at random.

The 16 selected lines were also put through a second generation of conditioning to fertiliser treatment.

Subsequent experiments in this series, involving seed from plants which had had one generation of conditioning followed by a generation of growth under uniform conditions and from plants which had received two successive generations of conditioning, were designed on a basis similar to that outlined above, with only minor variations in the number of lines and replications used. 
For the experiments combining fertiliser and photoperiodic treatments, an All or NoNe fertiliser treatment was used in all four possible combinations with short or long day-length ( 8 and 16 hours respectively). Two strains were used in the initial treatments. From each of these four environments three lines per strain were selected for further experiments. This seed and the control seed obtained from the original strains were subjected to the treatment for breaking dormancy. Each of the 24 lines (12 per strain) was used twice in any one replicate, once with and once without fertiliser,

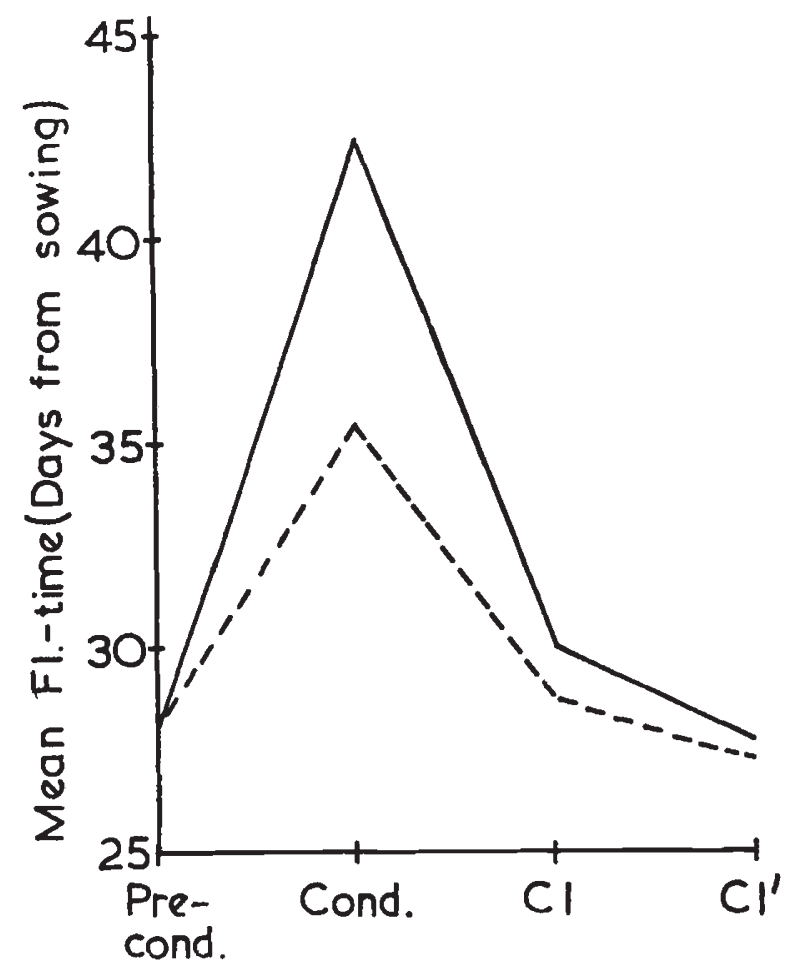

\section{GENERATION}

FIG. 1.-Relationship between flowering-time and generation of conditioning for the Langridge strain of $A$. thaliana. The solid line represents the mean flowering-time of the group which did not receive fertiliser, the broken line the mean flowering-time of the group which did receive fertiliser. Generations used were plants prior to treatment, during treatment and $\mathrm{I}$ and 2 generations removed from treatment respectively.

whilst for the controls, 3 from each strain were grown in each of the 2 fertiliser treatments per replicate. The experiment, which was of a randomised block design with five replicate-blocks, was laid down in a glasshouse. Supplementary light was provided such that all plants received 16 hours of light a day. The whole experiment was surrounded by a guard row.

The results obtained from these experiments indicated that neither of the two sets of treatments applied produced any permanent effect in $A$. thaliana. There was a decrease in the differences between treatment groups which was correlated with the number of generations of growth under unitorm conditions (fig. I). This decrease was found to be more rapid for differences attributable to light than for those produced by fertiliser 
alone. Transmission of effect from parent to the early stages of the growth of its offspring, which was thought to be due to the operation of a mechanism affecting the maternal nutrition of the seed, could be seen from data on growth-rates, where differences between groups at any one time could be related to differences in emergence, and from the establishment of a positive correlation between flowering-time of parent and mean germination-time of offspring despite the application to the seed of a treatment to break dormancy.

It is clear from the results that, within the specific experimental conditions described, the system of inheritance operating in Durrant's flax was not demonstrable in $A$. thaliana. There may be various reasons for this failure. In particular, it is possible that, because $A$. thaliana is an ephemeral plant and therefore makes only modest demands on its external environment, the range of treatments employed were not drastic enough to produce any permanent detrimental effect. Although no permanent effect was produced, it was possible to condition the plant and produce differences which persisted for two generations (see fig. I). This, therefore, seems to indicate that a system similar to Dauermodification was operating. Turning to plants with a longer life-cycle, it is possible that Durrant's success with flax may be due at least in part to the fact that this plant is relatively unimproved. For this reason experiments are now proceeding in an attempt to condition an inbred line of Nicotiana rustica, a species about which a considerable amount of information exists regarding the inheritance of variation under major genic or polygenic control.

Acknowledgments. - I wish to express my thanks to Professor K. Mather, F.R.s., and to Dr J. L. Jinks for their helpful advice and encouragement throughout.

This work has been undertaken whilst in receipt of an Agricultural Research Council Studentship.

\section{REFERENCES}

DURRANT, A. 1958. Environmental conditioning of flax. Nature, 181, 928-929. DURrant, A., AND TYSON, H. I96o. Conditioned lines of flax. Nature, 185, 60 . JOHANNSEN, W. 1909. Elemente der exakten Erblichkeitslehre. Jena.

LANGridge, J. L. I957. The aseptic culture of Arabidopsis thaliana. Aust. F. Biol. Sci., $10,243-252$. 